Accelerator Department BROOKHAVEN NATIONAL LABORATORY Associated Universities, Inc. Upton, New York 11973

AGS Division Technical Note . $\mathrm{NO}_{3} .207$

\title{
RESONANCE JUMPING OF POLARIZED PROTONS
}

S.Y. Lee, S. Tepikian, E.D. Courant

Sep tember 4,1984

\section{Abstract}

Spin transfer matrix method is applied to calculate the proton polarization of resonance jumping. Our results agree well with the experimental data of AGS. Problems and possible improvements of the resonance jumping are discussed. 1. Introduction

Polarized proton beam becomes an important tool for the study of the fundamenta1 interaction between protons. Unfortunately there are a number of resonances of the accelerator, which could depolarize particles of the beam. To maintain the polarization of the particles during the acceleration through the resonance, the vertical tune is shifted by a set of quadrupoles. This method is called resonance jumping. ${ }^{1-3}$ There are theoretical calculations of resonance jumping based on pertubation expansion.1,4 Unfortunate1y, the calculation is only valid for $|\varepsilon|^{2} / \alpha \ll 1$, where $\varepsilon$ is the resonance strength, and $\alpha$ is the beam particle acceleration rate. However, many resonances have the property that $|\varepsilon|^{2} / \alpha \sim 1$.

There are several experimental data available at present; it is therefore timely to calculate the polarization of the beam with the tune shift near resonance. Recently, we have developed a spin transfer matrix method to 
calculate the polarization of the beam particles. 5 Using this method, we can calculate the polarization of the beam with resonance jumping easily, provided that the tune shift can be modeled as a linear function of time or particle rotational angle in the accelerator. In fact, this simple tune shift model is very close to the experimental reality. ${ }^{3}$ In this short note, we aim to study the physics of the resonance jumping and the geometrical picture. Method of calculation shal1 be discussed fully in ref. 5. In Section 2, we present the mode1 and discuss the result of resonance jumping. Section 3 discusses the calculation of resonance jumping including slow quadrupoles. Conclusion and discussion are given in Section 4.

2. Polarization with a Simple Tune-shift Mode1

A resonance jump is obtained by firing a special set of quadrupoles, which shift the tune of the machine upward or downward suddenly and decay approximately linearly so that the tune returns to its nominal value within a characteristic time $\Delta t$. This tune shift can be represented by a simple model for resonance tune shift $\Delta(k P \pm v)$ as

$$
\Delta(k P \pm \nu)= \begin{cases}-\Delta v+\alpha_{\nu}\left(\theta-\theta_{1}\right) & \theta_{2} \geq \theta \geq \theta_{1} \\ 0 & \text { elsewhere }\end{cases}
$$

where $\alpha_{v}=\Delta v /\left(\theta_{2}-\theta_{1}\right)=\Delta v /(\omega \Delta t)$ corresponds to decay slope of the jumping quadrupoles. At $A=A_{1}$, the quadrupoles were fired. At $A=A_{2}$, the tune of the machine is back to its nominal value.

Let us assume further that the particle is accelerated unifomly with

$$
\gamma G=(\gamma G)_{\text {resonance }}+\alpha \theta
$$


where $\alpha$ is the acceleration rate. For AGS at BNL, $\alpha=4.86 \cdot 10^{-5}$ with $\theta$ measured in radian, which corresponds to $160 \mathrm{keV}$ energy gain per turn for proton. We assume that $\alpha_{\nu}$ to be somewhat smaller than $\alpha$.

By assuming the linear tune shift, the spin precession equations developed by Froissart and Stora, ${ }^{6}$ and Courant and Ruth ${ }^{4}$ can be solved analytically in terms of confluent hypergeometric functions by using spin transfer matrix method. 5

The upper part of Fig. 1 shows the experimental data obtained by AGS data group ${ }^{3}$ by measuring the polarization at momentum $p=10.3 \mathrm{GeV} / \mathrm{c}$ as a function of quadrupole firing time (old Gauss clock). The lower part of Fig. 1 shows our theoretical calculation for $\gamma G=\nu$ resonance with

$$
\begin{aligned}
& \Delta \nu=.2 \\
& \Delta t=2.5 \mathrm{~ms} \\
& \varepsilon=-.007862-i .00004
\end{aligned}
$$

where $\varepsilon$ is strength of the resonance for $\gamma^{G}=\nu$ calculated by program DEPOL ${ }^{4}$ with normalized emittance $\varepsilon_{\mathrm{N}}=8.03$ mmmad $^{7}$ and initial polarization $60 \%$.

We observe in Fig. I that there are three distinctive regions. Region I has the feature with flat plateau of negligible depolarization. Here the resonance is indeed successfully jumped. Region II has the feature of spin flip. This is because the effective acceleration rate of particle acceleration through resonance is $\alpha-\alpha_{\nu}$, which is much smaller than $\alpha$. Thus the dimensionless resonance strength $\pi|\varepsilon|^{2} / 2\left(\alpha-\alpha_{v}\right)$ is much larger. Region III appears to be interesting side-peaking.

These three regions can be separated by three characteristic "times", $0, \theta_{a}$ and $A_{b}$, indicated on Fig. $1 ;$ Fig. 2 shows the schematic quadrupole firing for 
$\theta_{1}=\theta_{a} ; \theta_{1}=\theta_{b}$ and $\theta_{1}=0$ respectively. We see easily that Region I corresponds to the firing time $A_{a} \leq \theta_{1} \leq 0$ and Region II corresponds to $\theta_{b} \leq \theta_{1}$ $\leq \theta_{a}$, where the effective acceleration rate is $\alpha-\alpha_{v}$. From Fig. 2 , we find easily that

$$
\begin{aligned}
& \left|\theta_{a}\right|=\Delta v / \alpha \\
& \left|\theta_{a}-\theta_{b}\right|=\Delta v\left(\frac{1}{\alpha_{v}}-\frac{1}{\alpha}\right)
\end{aligned}
$$

Thus the width of spin-f1ip region will be smaller for smaller $\alpha-\alpha_{v} 0$

The physics of Region III is least understood. The oscillatory structure in Fig. $1 b$ is due to interference within the asymptotic region of the resonance 5 $\left(\theta_{2} \simeq 500 \mathrm{rad}\right.$ away from the resonance). But the reason that the side peak of Fig. la appears to be the envelope of the oscillatory structure in Fig. 1b is not clear to us. Note also that our resonance strength appears to be somewhat too strong in comparison with the data. Fig. 3 illustrates the fact that when the resonance strength becomes weaker the envelope of side peak also becomes larger in Region III, while the spin-flip strength is also decreased in Region II.

3. Slow Jump

To obtain an edge of larger $\Delta \nu$ jumping without crossing integer or half integer tunes, slow quadrupoles are set up to increase the tune of the machine in the time scale of $10 \sim 20 \mathrm{msec}$. Within this time the fast quadrupoles are fired with time constant to be $2.5 \mathrm{msec}$ ( ily to calculate the polarization of this tune jump. We find no advantage for improving polarization. However, if the nominal tune is too close to an integer or half integer, then the slow quadrupole is indeed required to give room for fast quadrupole performing its tune shifting. From our study, we found that the 
most favorable condition is shown in Fig. 4. Fig. 5 shows the degree of polarization with the following parameters,

$$
\begin{aligned}
& |\varepsilon|=0.0138 \\
& \Delta t=2.5 \mathrm{~ms} \\
& \Delta t_{s}=10 \mathrm{~ms}
\end{aligned}
$$

with the assumption of initial polarization to be $60 \%$. The strength corresponds to the 36-v resonance of AGS. We note that there are two flat-top regions, which correspond to jumping through resonance via $\Delta \nu_{s}$ and $\Delta \nu$ respectively. Based on the same geometrical argument of eq. (4), the spin flip region can be described by

$$
\Delta \theta_{\text {flip }}=\Delta \nu\left(\frac{1}{\alpha_{s}+\alpha_{v}}-\frac{1}{\alpha}\right),
$$

where $\alpha_{s}$ and $\alpha_{v}$ are the slopes of slow tune shift and fast tune shift respectively. Eq. (5) implies that the spin flip region of Fig. 5 will be narrower than that of Fig. 1 .

Fig. 5 also shows the double-hump structure which is observed by the AGS data group for $36-v$ resonance. Since the strength of this resonance is large, the polarization of the beam will be preserved by spin flip without resonance jump. This is seen also on Fig. 5 for $A<-7000$ rad or $\theta>3000$ rad.

4. Conclusion and Discussion

In conclusion, we have studied the resonance jumping for polarized beam based on a simple model of quadrupole tune shift. Results of our calculation fit fairly well with the experimental result of polarization measurement. The polarization obtained can be understood in terms of geometrical picture. The width of successful resonance jumping is $\Delta \nu / \alpha$.

Our theoretical calculation is based on a simple model of jumping without considering the emittance blow up through the quadrupole firing. Non-adiabatic 
change in tune gives rise to a mismatch in the phase advance and the emittance will grow through tune jump. Fig. 6 shows the emittance measured in AGS in the processes of quadrupole firing. Our model does not take into account the emittance growth at the time of resonance crossing. We do not understand fully the mechanism for the emittance blow up. This is a rather interesting question of its own right in accelerator physics. Since strength of resonance increases with square root of the emittance, understanding the emittance blow up is important to accelerate the polarized beam to higher energies.

To control the emittance growth, ${ }^{8}$ one can also use a smaller tune shift for these resonances with moderate resonance strength. As an example, the tune shift for the first resonance in Fig. 1 can be chosen to be $\Delta v=0.1, \Delta t=1.25$ msec, provided that the tune of the machine is measured to the accuracy of $\simeq 2 * 10^{-2}$. However, adiabatic crossing of resonances with strength $|\varepsilon| \gg 1 * 10^{-2}$ (or $92 \%$ spin flip adiabatic crossing with acceleration rate $\alpha=4.86 \cdot 10^{-5}$ ) to obtain a respectable amount of spin flip without firing resonance jump quadrupoles (to control the emittance growth) is a preferred procedure. Since the strength of the spin resonances depends on the emittance of the beam, it is interesting to $\operatorname{cool}$ the beam emittance in the proposed accumulator/ booster ring. ${ }^{9,10}$ The cooling procedure is more efficient in the lattice with separated regions of large dispersion and small dispersion respectively. The effect of cooling to the spin polarization is not yet well understood. Small scale experiments on this problem should yield interesting results. Acknow ledgment

We are grateful to have discussions with Y.Y. Lee, L.G. Ratner, L. Ahrens, E. Gill, and H.W.J. Foelsche on the experimental aspects of polarized proton beams. 


\section{Figure Captions}

Fig. 1. The experimenal polarization is shown as a function of the quadrupole firing time in the upper part of the figure obtained by the AGS data group (see ref. 3). Theoretical calculation of a simple quadrupole jumping model is shown below (see text for parameters).

Fig. 2. Representation of simple tune-jump model for the boundaries of Regions I, II, and III shown in Fig. 1.

Fig. 3. Calculated polarization of the beam as a function of quadrupole firing time with different resonance strength as indicated on the figure. A11 other parameters of this calculation are the same as that of Fig. 1.

Fig. 4. Schematic representation of a slow quadrupole firing plus a fast quadrupole firing. The characteristic times of the slow quad and fast quad are $10 \mathrm{~ms}$ and $2.5 \mathrm{~ms}$, respective $1 \mathrm{y}$.

Fig. 5. Calculated polarization as a function of the fast quad firing "time" $\theta$. The slow quad was fired $7.5 \mathrm{~ms}$ earlier, $\Delta \nu_{s}=0.1$ and $\Delta v=0.2$. The characteristic time for slow quad and fast quad are $10 \mathrm{~ms}$ and $2.5 \mathrm{~ms}$ respectively. All other parameters remain the same.

Fig. 6. Real time and the emittance of the beam as a function of Gauss clock are shown on the figure. The data is taken from ref. 7 
References

1. T. Khoe, R.L. Kustom, R.L. Martin, E.F. Parker, C.W. Potts, L.G. Ratner, R.E. Timm, A.D. Krisch, J.B. Robert, and J.R. O'Fallon, Particle Accelerator $\underline{6}, 213(1975)$.

2. E. Grorud, J.L. Laclare, G. LeLeux, A. Nakach, and A. Ropert, AIP Conference, Proc. No. 95, ed. G.M. Bunce, p 407 (1982).

3. L.G. Ratner and AGS data group, private communication.

4. E.D. Courant and R.D. Rath, BNL-51270 (1980).

5. S. Tepikian, S.Y. Lee, and E.D. Courant, to be published.

6. M. Froissart and R. Stora, Nuc 1. Inst. and Methods, 1, 297 (1960).

7. L. Ahren and E. Gill, private communication.

8. E.D. Courant, to be published.

9. Accumulator/Booster Proposal for the AGS, BNL 32949-R.

10. P. Bambade, J. Buon, and S. Tepikian, BNL-34350, HEF84-1 . 


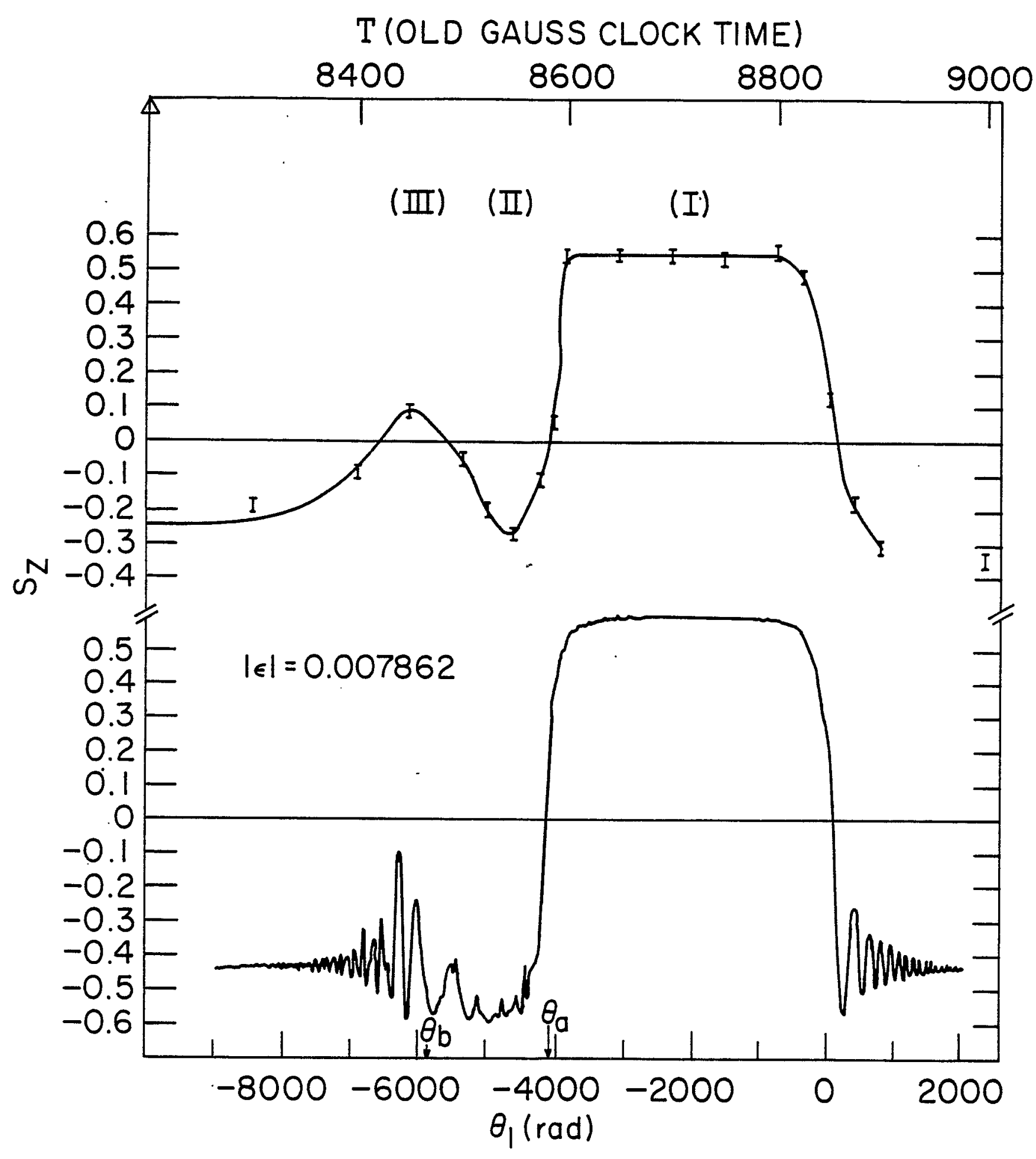

fig. 1 


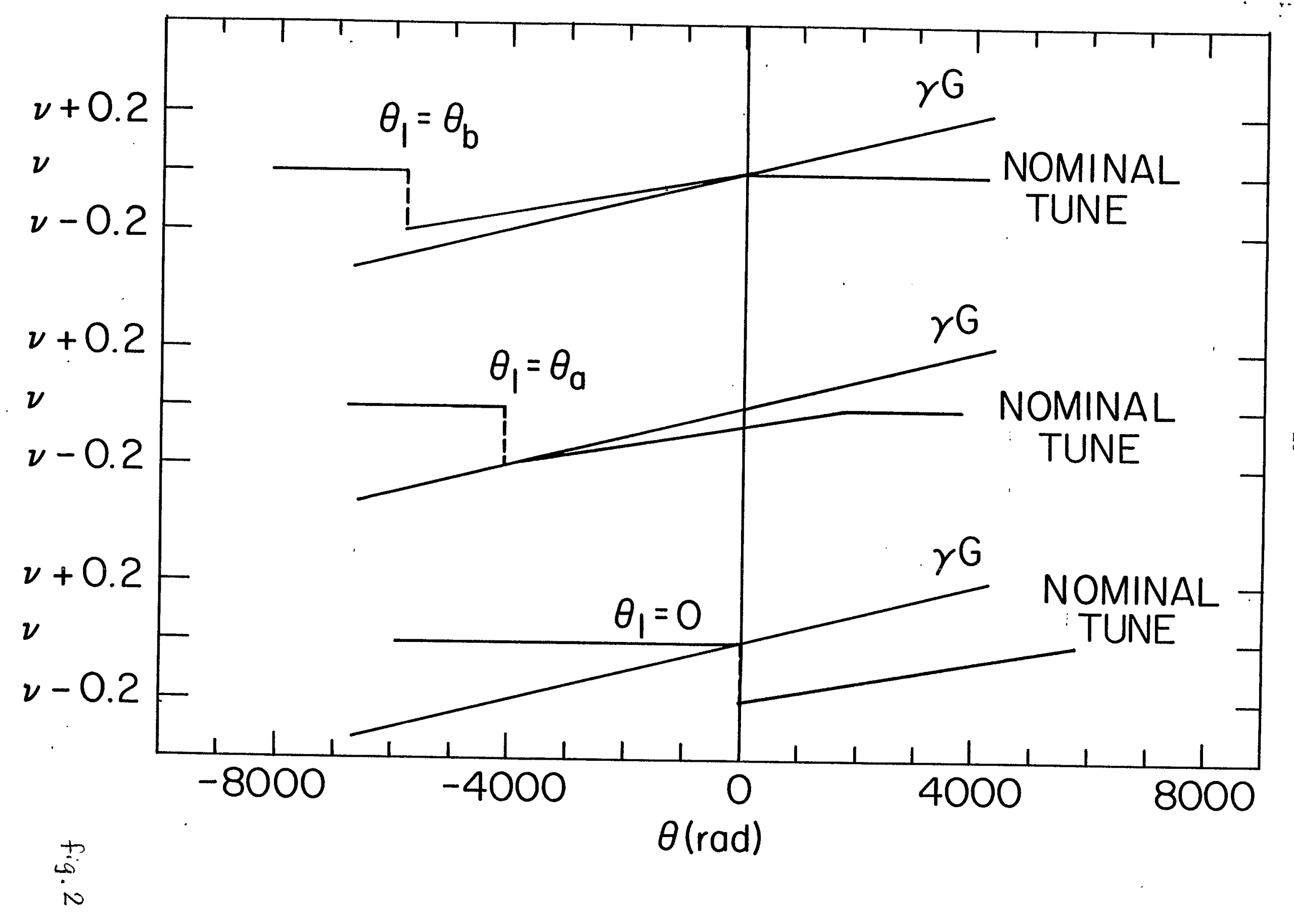




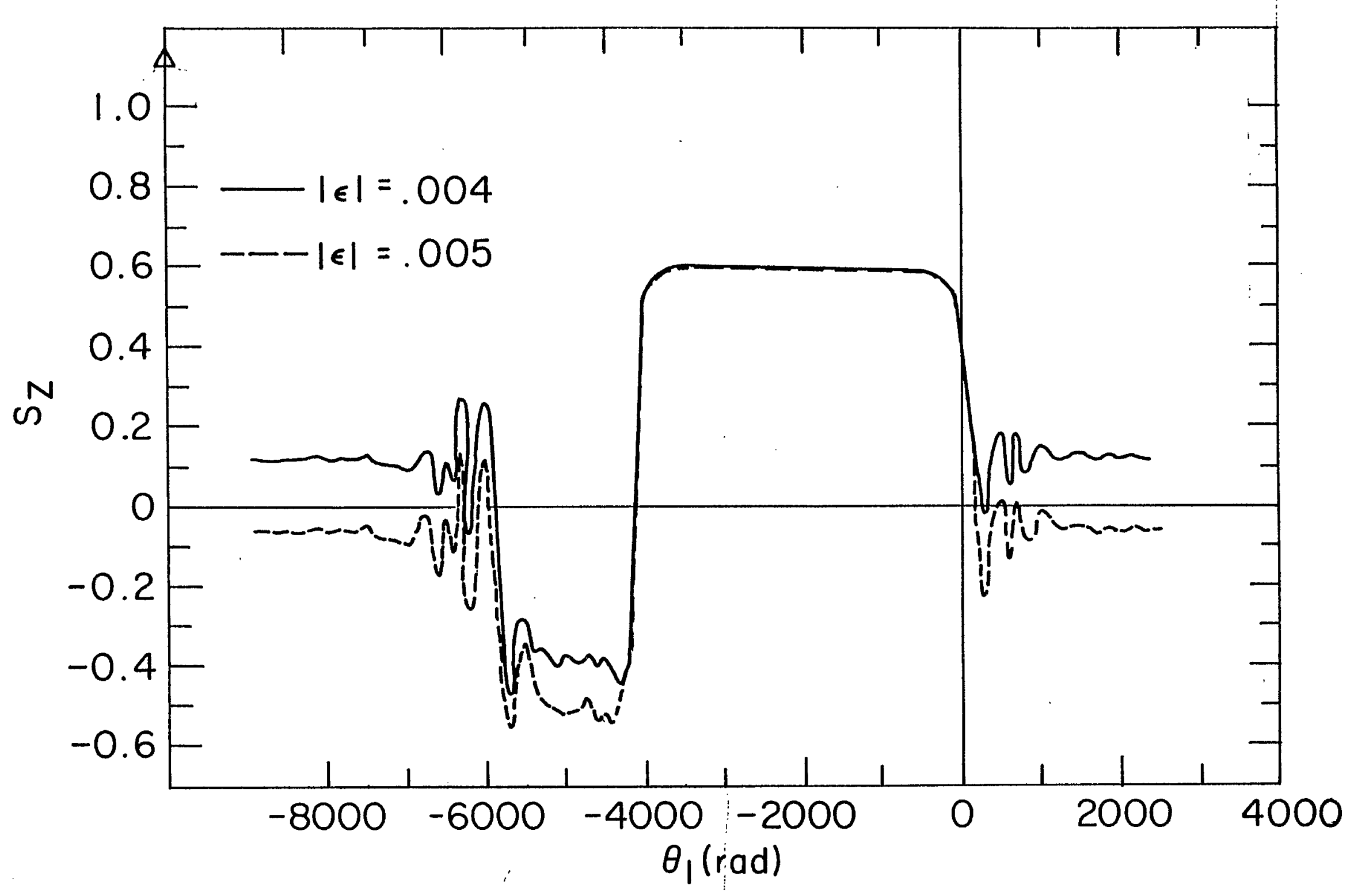

$\underset{\omega}{ \pm}$ 


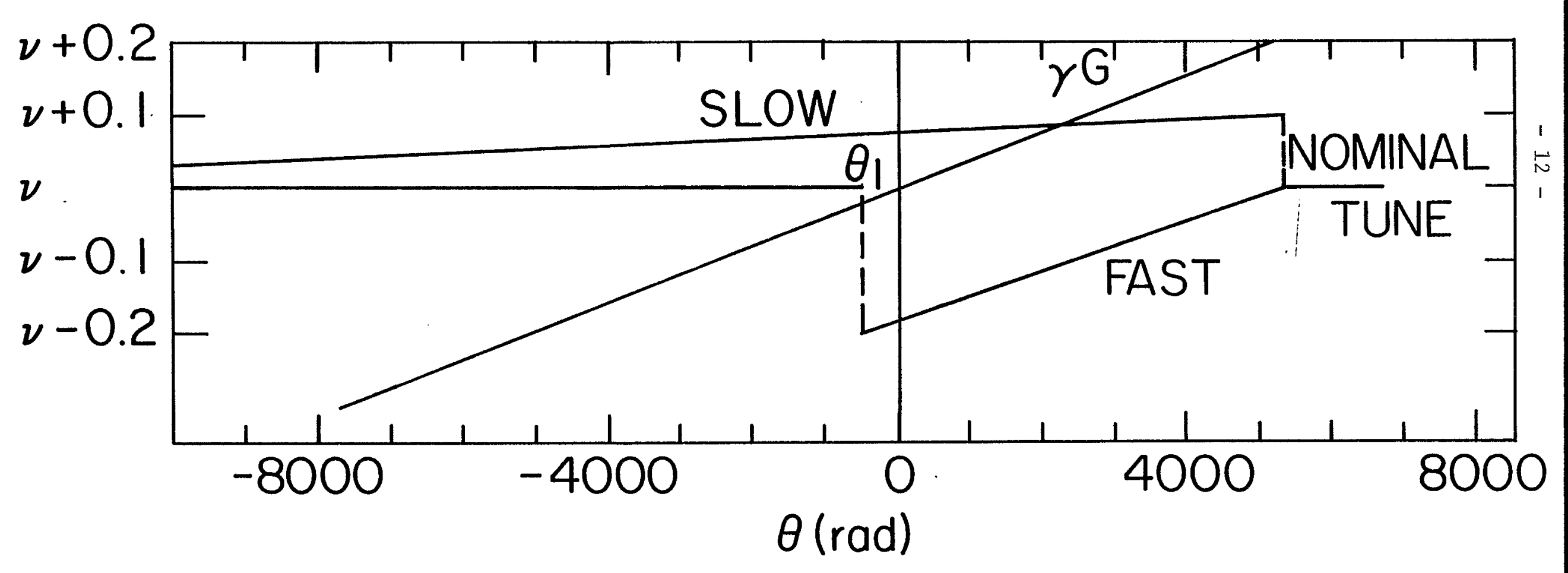

$t$
+ 


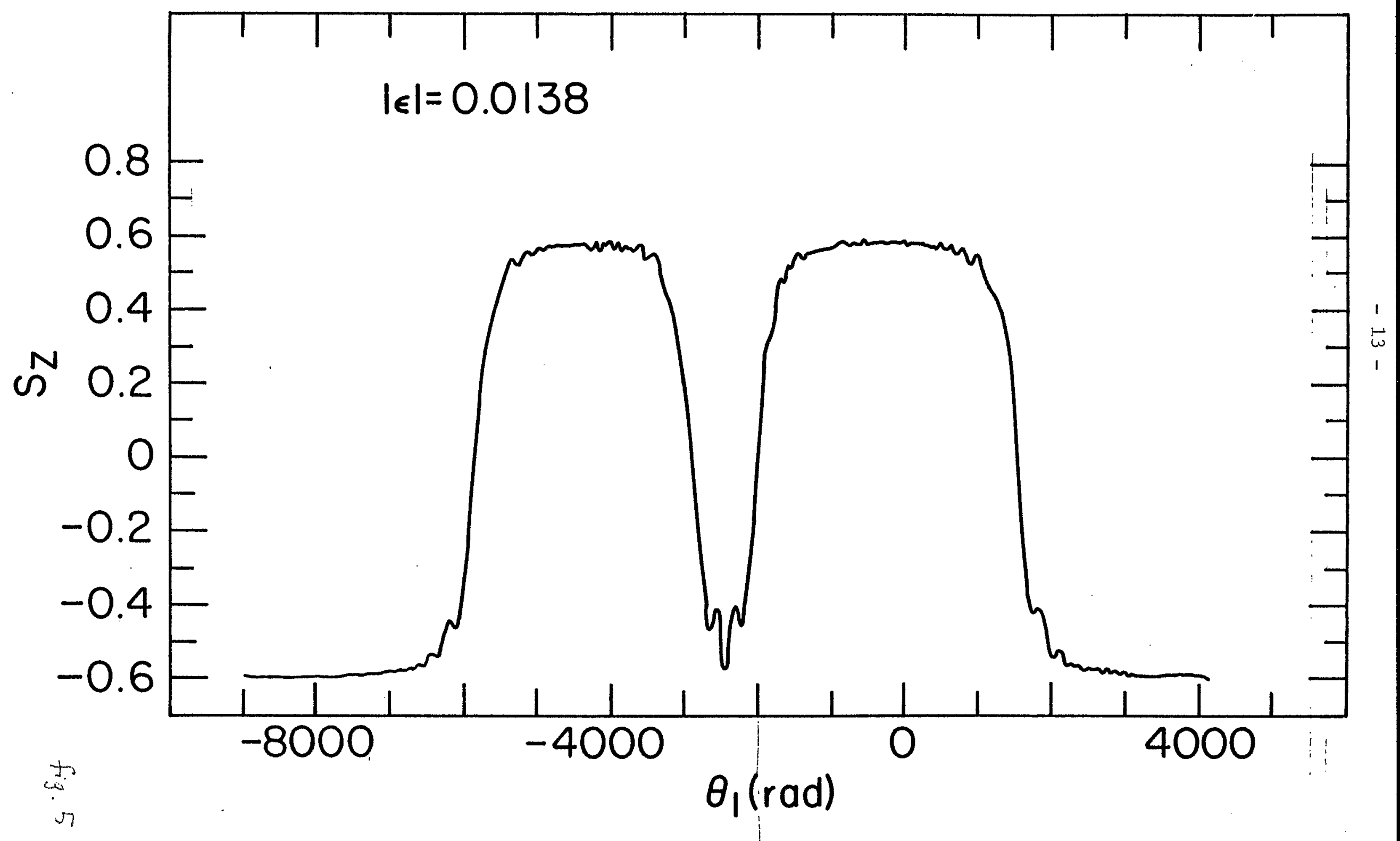




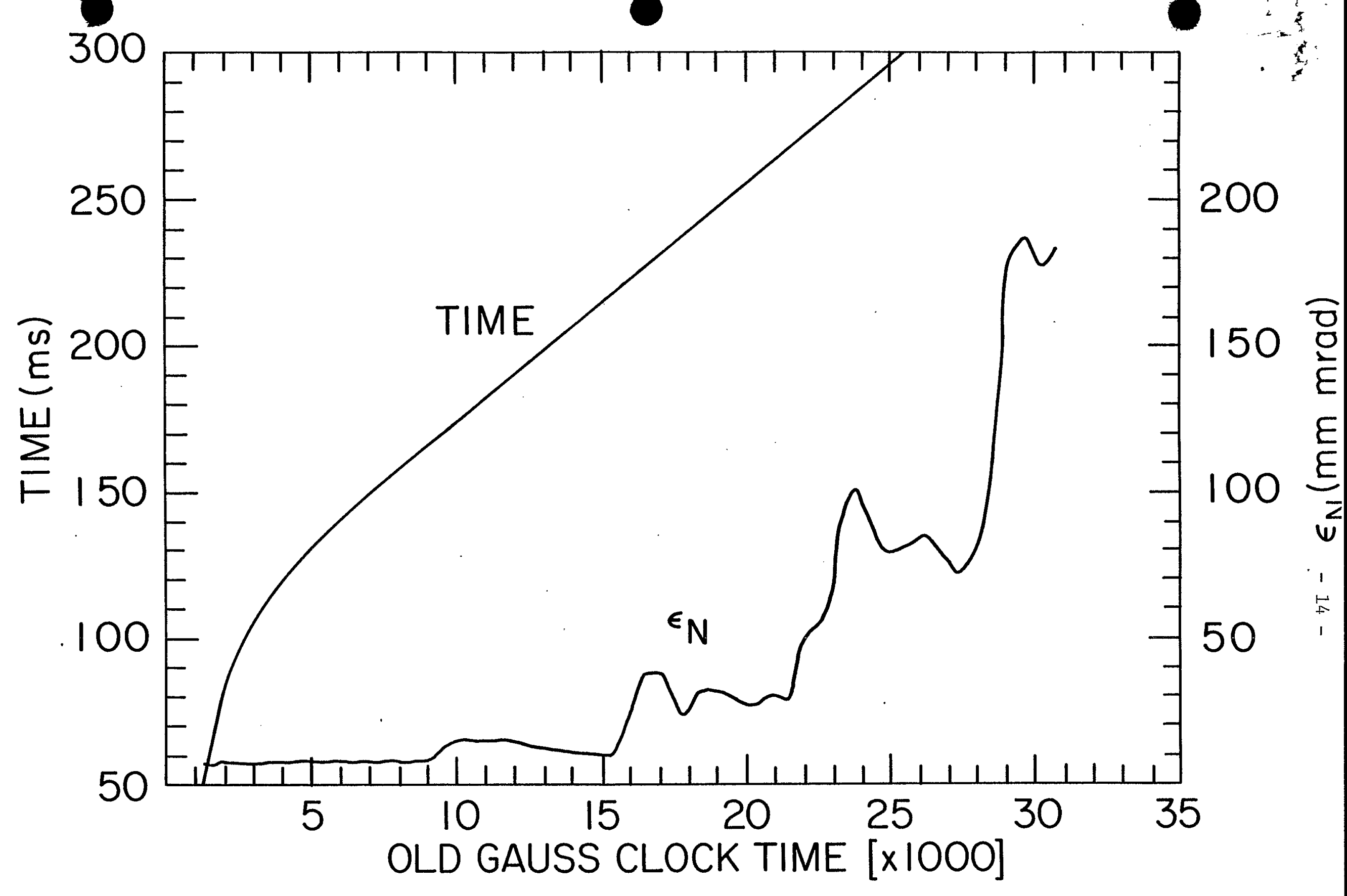

